\title{
Class Groups of Complex Quadratic Fields
}

\author{
By R. J. Schoof
}

\begin{abstract}
We present 75 new examples of complex quadratic fields that have 5-rank of their class groups $\geqslant 3$. Only one of these fields has 5 -rank of its class group $>3$ : The field $\mathbf{Q}(\sqrt{-258559351511807})$ has a class group isomorphic to
\end{abstract}

$$
C(5) \times C(5) \times C(5) \times C(5) \times C(2) \times C(11828) .
$$

The fields were obtained by applying ideas of J. F. Mestre to the 5-isogeny $X_{1}(11) \rightarrow X_{0}(11)$.

1. Introduction. For any, multiplicatively written, finite abelian group $A$ and any prime $p$, we define the $p$-rank of $A, d_{p} A=\operatorname{dim}_{\mathbf{F}_{p}} A / A^{p}$ : the number of generators of the $p$-primary part of $A$. For any number field $K$, we denote by $\Delta(K)$ its absolute discriminant and by $\mathrm{Cl}(K)$ its ideal class group: a finite abelian group. The cyclic group of order $n$ is denoted by $C(n)$. In the past decade some effort has been made to construct complex quadratic fields $K$ with large $d_{p} \mathrm{Cl}(K)$ for odd prime $p$. Many examples of class groups with 3-rank $=3$ and 3-rank $=4$ have been found by Shanks and others [2], [3], [9] and, fairly recently, Solderitsch [10] gave examples of complex quadratic fields $K$ with $d_{5} \mathrm{Cl}(K)=3$, and one example with $d_{7} \mathrm{Cl}(K)=3$. Also, in [4] Diaz y Diaz gave an example of a complex quadratic field $K$ with $d_{5} \mathrm{Cl}(K)=3$, that has a comparatively small discriminant. In this paper we present 74 complex quadratic fields $K$ with 5-rank of their class groups equal to 3 and one example with 5-rank of its class group equal to 4 . We obtained these examples by computing the class groups of 356 complex quadratic fields; the discriminants of these fields are parametrized by an 8th-degree polynomial $M(t) \in \mathbf{Z}[t]$.

2. The Polynomials $M(t)$. In this section we will explain the construction of polynomials, $M(t) \in \mathbf{Z}[t]$, that we use to parametrize a series of complex quadratic fields with class groups having $p$-rank $\geqslant 2$, for some prime $p$. The ideas involved are due to J. F. Mestre and are in [5]; here we only give the formulae to compute the polynomials $M(t)$.

Let $p$ be a prime and $F$ an elliptic curve defined over $\mathbf{Q}$ with a $\mathbf{Q}$-rational point $P$ of order $p$ on it. By $E$ we denote the elliptic curve $F /\langle P\rangle$, which is, again, defined over $\mathbf{Q}$. We denote the isogeny $F \rightarrow E$ by $\varphi$. Let $Q$ be a point on $E$ with coordinates in some algebraic number field $K$. Then the coordinates of the points in $\varphi^{-1}(Q)$ generate an extension of $K$ that is unramified over $K$ and cyclic of degree $p$, provided that $Q$ is submitted to certain conditions, cf. [5, Proposition II.1.3 and Proposition II.3.3]. To obtain quadratic fields with class groups having a $p$-rank $\geqslant 2$, one tries to find two distinct points $Q_{1}$ and $Q_{2}$ on $E$ with coordinates in a quadratic number

Received March 23, 1981.

1980 Mathematics Subject Classification. Primary 12-04, 12A25, 14 K07. 
field $K$; under certain conditions, the points in the fibres $\varphi^{-1}\left(Q_{1}\right)$ and $\varphi^{-1}\left(Q_{2}\right)$ generate two independent unramified cyclic extensions of degree $p$ of $K$, which, by class field theory, implies that $C(p) \times C(p)$ is a quotient of $\mathrm{Cl}(K)$, whence $d_{p} \mathrm{Cl}(K) \geqslant 2$.

Assume that the curve $E$ is given by an equation:

$$
Y^{2}=X^{3}-\frac{c_{4}}{48} X-\frac{c_{6}}{864}, \quad c_{4}, c_{6} \in \mathbf{Z} .
$$

Let $Q_{1}=\left(\xi_{1}, \eta\right)$ and $Q_{2}=\left(\xi_{2}, \eta\right)$ be two distinct points on the curve $E$ with $\xi_{1}$, $\xi_{2} \in \mathbf{Q}$ and, as a consequence, $\eta$ in some quadratic number field. We wish to compute the field $\mathbf{Q}(\eta)$, which will play the role of the field $K$ from above. We have that

$$
\eta^{2}=\xi_{1}^{3}-\frac{c_{4}}{48} \xi_{1}-\frac{c_{6}}{864}=\xi_{2}^{3}-\frac{c_{4}}{48} \xi_{2}-\frac{c_{6}}{864}
$$

so

$$
\xi_{1}^{2}+\xi_{1} \xi_{2}+\xi_{2}^{2}=\frac{c_{4}}{48}
$$

Let $\zeta$ denote a primitive sixth root of unity. Then $\zeta$ satisfies $\zeta^{2}-\zeta+1=0$. Put

$$
\theta=\frac{12}{1+\zeta}\left(\xi_{1}+\xi_{2} \zeta\right) \in \mathbf{Q}(\zeta)
$$

Then $\operatorname{Norm}(\theta)=c_{\mathbf{4}}$, and one easily computes

$$
\theta^{3}+\bar{\theta}^{3}=12^{3} \xi_{1} \xi_{2}\left(\xi_{1}+\xi_{2}\right) .
$$

It follows that

$$
\eta^{2}=\xi_{1}^{3}-\frac{c_{4}}{48} \xi_{1}-\frac{c_{6}}{864}=-\xi_{1} \xi_{2}\left(\xi_{1}+\xi_{2}\right)-\frac{c_{6}}{864}=-\frac{\bar{\theta}^{3}+\theta^{3}}{12^{3}}-\frac{c_{6}}{864} .
$$

So

$$
(72 \eta)^{2}=-3\left(\theta^{3}+\bar{\theta}^{3}+2 c_{6}\right)
$$

and the field $\mathbf{Q}(\eta)$ can be written as

$$
\mathbf{Q}(\eta)=\mathbf{Q}\left(\sqrt{-3 \operatorname{Trace}\left(\theta^{3}+c_{6}\right)}\right) .
$$

From this representation of $\mathbf{Q}(\eta)$ it is plain that the numbers $\theta, \zeta^{2} \theta, \zeta^{-2} \theta, \bar{\theta}, \zeta^{2} \bar{\theta}$, $\zeta^{-2} \bar{\theta}$ all give the same field $\mathbf{Q}(\eta)$.

Next we parametrize the conic (1), and we obtain a parametrization of the family of fields $\mathbf{Q}(\eta)$. To make sure that the equation (1) describes a nonempty curve over $\mathbf{Q}$, we will make the assumption that $c_{4}$ is a norm of an element of $\mathbf{Q}(\zeta)$. The numbers $\theta$ with $\operatorname{Norm}(\theta)=c_{4}$ can be parametrized, e.g., by

$$
\theta(t)=(a+b \zeta)\left(\frac{2 t+1}{t^{2}+t+1}+\frac{t^{2}-1}{t^{2}+t+1} \zeta\right), \quad t \in \mathbf{Q} \cup\{\infty\},
$$

where $a, b \in \mathbf{Z}$ and $a+b \zeta$ is a fixed number such that $\operatorname{Norm}(a+b \zeta)=c_{4}$, i.e., $a^{2}+a b+b^{2}=c_{4}$. 
Substituting (3) in (2) eventually gives us that $\mathbf{Q}(\eta)=\mathbf{Q}(\sqrt{M(t)})$ with $M(t) \in \mathbf{Z}[t]$ of degree 8 . The polynomial $M(t)$ can be computed as follows: Let

$$
\begin{array}{lll}
\alpha=-a+b, & \mu_{0}=\nu_{1}-2 c_{6}, \\
\beta=-a-2 b, & \mu_{1}=-2 \nu_{3}-6 c_{6}, \\
\gamma=2 a+b, & \mu_{2}=5 \nu_{4}-12 c_{6}, \\
\nu_{1}=\alpha \beta \gamma, & \mu_{3}=-2 \nu_{2}-14 \nu_{1}-14 c_{6}, \\
\nu_{2}=\alpha^{3}+\beta^{3}+\gamma^{3}, & \mu_{4}=5 \nu_{3}-12 c_{6}, \\
\delta_{3}=\alpha^{2} \beta+\beta^{2} \gamma+\gamma^{2} \alpha, & \mu_{5}=-2 \nu_{4}-6 c_{6}, \\
\nu_{4}=\alpha^{2} \gamma+\beta^{2} \alpha+\gamma^{2} \beta, & \mu_{6}=\nu_{1}-2 c_{6} .
\end{array}
$$

Then

$$
M(t)=3\left(t^{2}+t+1\right) \sum_{i=0}^{6} \mu_{i} t^{i} \in \mathbf{Z}[t] .
$$

Of course, $M(t)$ depends upon the choice of the number $a+b \zeta$. If $t \in \mathbf{Q} \cup\{\infty\}$, then the rational numbers (or $\infty$ )

$$
t,-1-\frac{1}{t},-\frac{1}{t+1}, \frac{-b t+a}{(a+b) t+b}, \frac{a t+(a+b)}{b t-a},-\frac{(a+b) t+b}{a t+(a+b)}
$$

all give, up to a square, the same value for $M(t)$, i.e. these numbers give the same field $\mathbf{Q}(\eta)$.

In order to insure that the class group of $\mathbf{Q}(\eta)$ has $p$-rank $\geqslant 2$, one submits the points $Q_{1}$ and $Q_{2}$ to certain conditions (cf. [5, Proposition II.2.2]). Numerical experience suggests that we should only bother about one of these:

(4) "the points $Q_{1}$ and $Q_{2}$ should not become singular modulo any prime of $K$."

This condition boils down to simple congruence conditions on $t$ modulo primes that divide the conductor of $E$.

In the next section we use the formulae given above to obtain quadratic fields having class groups with $p$-rank $\geqslant 3$, for $p=5$. At present, it seems unclear why such a large fraction of the computed class groups has a 5-rank greater than 2 .

3. The Computations. We apply the formulae from Section 2 to the 5-isogeny $X_{1}(11) \rightarrow X_{0}(11)$. An equation for $X_{0}(11)$ can be found in [6, p. 82]:

$$
Y^{2}+Y=X^{3}-X^{2}-10 X-20
$$

So, in the notation of $[6$, p. 36], we have that

$$
\left(a_{1}, a_{2}, a_{3}, a_{4}, a_{6}\right)=(0,-1,1,-10,-20),
$$

whence (in the notation of $\left[6\right.$, p. 36]) $c_{4}=496$ and $c_{6}=20008$. Now $c_{4}$ is a norm from $\mathbf{Q}(\zeta)$, e.g. Norm $(20+4 \zeta)=c_{4}$, and we take

$$
a=20 \text { and } b=4 \text {. }
$$

A straightforward computation, using the formulae given in the previous section, gives us that, up to a square,

$$
M(t)=-\left(t^{2}+t+1\right) \cdot\left(47 t^{6}+21 t^{5}+598 t^{4}+1561 t^{3}+1198 t^{2}+261 t+47\right),
$$

which is, up to a linear transformation, Mestre's polynomial $m(t)$ in his Proposition II.2.2 in [5]. 
The conductor of $X_{0}(11)$ equals 11 and condition (4) here boils down to

$$
t \neq 2,-4,4(\bmod 11) \text {. }
$$

(i.e. if $t=p / q \in \mathbf{Q} \cup\{\infty\}$ with $p, q \in \mathbf{Z}, \operatorname{gcd}(p, q)=1$, then $p \neq 2 q,-4 q, 4 q$ $(\bmod 11))$.

We computed the class groups of the fields $\mathbf{Q}(\sqrt{M(t)})$ for $t \in \mathbf{P}_{1}(\mathbf{Q})$ with:

(i) $t$ satisfies (5).

(ii) $t=p / q ; p, q \in \mathbf{Z}$ with $|p|,|q|,|p+q| \leqslant 40$.

This comprised 356 nonisomorphic complex quadratic fields, and we obtained the following distribution of the 5-primary parts of their class groups:

\begin{tabular}{r|r|r|r|} 
5-primary part & freq. & 5-primary part & freq. \\
\hline$C(5)$ & 1 & $C(5) \times C(5) \times C(5)$ & 55 \\
$C(5) \times C(5)$ & 210 & $C(5) \times C(5) \times C(25)$ & 14 \\
$C(5) \times C(25)$ & 51 & $C(5) \times C(5) \times C(125)$ & 3 \\
$C(5) \times C(125)$ & 17 & $C(5) \times C(5) \times C(625)$ & 2 \\
$C(5) \times C(625)$ & 1 & $C(5) \times C(5) \times C(5) \times C(5)$ & 1 \\
$C(5) \times C(3125)$ & 1 & & \\
\hline total & 218 & total & 75
\end{tabular}

For the following 75 values of $t$, the field $\mathbf{Q}(\sqrt{M(t)})$ has 5-rank of its class group $\geqslant 3$ :

$$
\begin{aligned}
& \frac{1}{4}, \frac{1}{5}, \frac{7}{2}, \frac{2}{7}, \frac{7}{4}, \frac{7}{5}, \frac{1}{11}, \frac{11}{3}, \frac{3}{11}, \frac{2}{13}, \frac{5}{11}, \frac{7}{33}, \frac{11}{6}, \frac{2}{15}, \frac{5}{12}, \\
& \frac{11}{7}, \frac{16}{3}, \frac{11}{26}, \frac{17}{2}, \frac{14}{5}, \frac{7}{11}, \frac{10}{9}, \frac{5}{14}, \frac{6}{13}, \frac{15}{7}, \frac{1}{21}, \frac{2}{21}, \frac{23}{1}, \frac{19}{7}, \frac{12}{13}, \\
& \frac{17}{9}, \frac{7}{18}, \frac{20}{7}, \frac{11}{15}, \frac{16}{11}, \frac{11}{16}, \frac{20}{17}, \frac{17}{11}, \frac{20}{9}, \frac{27}{2}, \frac{1}{27}, \frac{18}{11}, \frac{9}{19}, \frac{16}{13}, \frac{25}{6}, \\
& \frac{3}{26}, \frac{24}{7}, \frac{5}{24}, \frac{19}{11}, \frac{11}{18}, \frac{8}{21}, \frac{9}{20}, \frac{1}{29}, \frac{28}{5}, \frac{21}{11}, \frac{1}{31}, \frac{20}{13}, \frac{32}{1}, \frac{19}{14}, \frac{17}{16}, \\
& \frac{2}{31}, \frac{33}{1}, \frac{33}{2}, \frac{23}{12}, \frac{11}{23}, \frac{29}{8}, \frac{27}{10}, \frac{12}{23}, \frac{35}{3}, \frac{34}{5}, \frac{2}{35}, \frac{1}{38}, \frac{14}{25}, \frac{39}{1}, \frac{11}{29} .
\end{aligned}
$$

(The values of $t$ are listed according to the size of the discriminants of the associated

\begin{tabular}{|c|c|c|c|c|c|c|c|}
\hline & $\Delta(\mathrm{K})$ & factorization & $t$ & $h$ & 5-part & rest & $I .(1, x)$ \\
\hline 1. & 18397407 & 3.7 .876067 & $1 / 4$ & $2(x)$ & $5>5>5$ & $2+8$ & 1.46 .5 \\
\hline 2. & $7777 \times 287$ & 31.103 .24359 & $1 / 5$ & $6(1)(x)$ & $5 \times 5 \times 5$ & $2 \times 24$ & 2.1 .37 \\
\hline 3. & 205996583 & 13.73 .131 .1657 & & $1(x)(x)$ & $5+5>25$ & $2 \times 2 \times 4$ & 2.189 \\
\hline$\overline{4}$. & 1156599359 & 47.67 .311 .1181 & $7 / 2$ & 34()() & $5 \times 5 \times 5$ & $2+2+68$ & 3.141 \\
\hline$\underline{S}$. & $2048(074559$ & 67.3323 .9199 & $2 / 7$ & 525()() & $5 / 5>5$ & $2+210$ & 3.644 \\
\hline$\overline{6}$. & 7558314879 & $3.31 .883 .92(041$ & $7 / 4$ & $60(0)(0)$ & $5 \times 5 \times 25$ & $2 \times 4 \times 12$ & 2.168 \\
\hline
\end{tabular}
fields. () course, every field occurs for at least six different values of $t$; we picked $t=p / q=0$ with $|p+q|$ minimal.) We list all 75 fields with their class groups in the table and single out 16 fields for special mention.

The following is a list of all complex quadratic fields $K$, with $d_{5}(\mathrm{l} l(K)=3$ and $|\Delta(K)|-1()^{10}$, known to us:

(Here and in the next table we denote by $n_{1} \times n_{2} \times \cdots \times n_{T}$ the abelian group $C\left(n_{1}\right) \times C\left(n_{2}\right) \times \cdots \times C\left(n_{T}\right)$.) Diaz y Diaz was the first to compute the class group of the fields $\underline{1}, \underline{2}$, and $\underline{3}$. The field $\underline{3}$ was found by him by an entirely different method [4]. 


\begin{tabular}{|c|c|c|c|c|c|c|c|}
\hline & $-\Delta(K)$ & factorization & $t$ & $h$ & 5-part & rest & $L(1, \chi)$ \\
\hline . & 47 & prime & ) & 5 & 5 & 1 & 2.291 \\
\hline$\underline{8}$. & 11199 & 3.3733 & 1 & 100 & $5 \times 5$ & 4 & 2.969 \\
\hline 9 . & 258559351511807 & 1171.1439 .153441403 & $14 / 25$ & 1478500 & $5 \times 5 \times 5 \times 5$ & $2 \times 11828$ & 2.889 \\
\hline 10. & 222637549223 & prime & $7 / 33$ & 434625 & $5 \times 5 \times 5$ & 3477 & 2.894 \\
\hline 11. & 3513582927119 & 487.7214749337 & $2 / 21$ & 2178000 & $5 \times 5 \times 5$ & $3 \times 5808$ & 3.650 \\
\hline 12. & 37262495315279 & 13.61 .46989275303 & $21 / 11$ & 7749000 & $5 \times 5 \times 5$ & $6 \times 10332$ & 3.988 \\
\hline 13. & 10368869999 & 97.106895567 & $3 / 8$ & 118750 & $5 \times 625$ & 38 & 3.664 \\
\hline 14. & 1449192975839 & 7.61 .163 .20821439 & $19 / 3$ & 1000000 & $5 \times 3125$ & $2 \times 2 \times 16$ & 2.610 \\
\hline 15. & 4574009420324 & $2^{2} .97 .2297 .5132209$ & - & 1088000 & $5 \times 5 \times 5$ & $2 \times 4 \times 1088$ & 1.598 \\
\hline 16. & 51887726858696 & $2^{3} .6485965857337$ & - & 4492500 & $5 \times 5 \times 5$ & $2 \times 215252$ & 1.959 \\
\hline
\end{tabular}

The field 7: $\mathbf{Q}(\sqrt{-47})$ occurs for $t=0$ (and $\left.t=-1, \infty, 5,-\frac{6}{5}, \frac{1}{6}\right)$; in the range of our computations it is the only value of $t$, satisfying the condition (5), for which the corresponding field has a class group with 5-rank $<2$. The field $\underline{8}: \mathbf{Q}(\sqrt{-11199})$ is the smallest field $K$ (small with respect to $|\Delta(K)|$ ) that has a class group whose 5 -rank equals 2 , cf. [1]. The next entry in our table, field $\underline{9}$, is the only example we found of a complex quadratic number field $K$ with $d_{5} \mathrm{Cl}(K)=4$. At present it is the only known example of a complex quadratic field possessing this property. We give four independent ideal classes of order 5 of this field $K$ by giving the associated reduced binary quadratic forms of discriminant $\Delta(K)$. Recall that a reduced binary quadratic form $a X^{2}+b X Y+c Y^{2}$ of discriminant $\Delta=b^{2}-4 a c$ corresponds to the ideal class

$$
\left\{\left(\mathbf{Z}+\frac{b+\sqrt{\Delta}}{2 a} \mathbf{Z}\right) \cdot \alpha: \alpha \in K^{\times}\right\}
$$

of $K=\mathbf{Q}(\sqrt{\Delta})$.

The four ideal classes correspond to:

$$
\begin{array}{r}
179988 X^{2}+55577 X Y+359138443 Y^{2}, \\
7536956 X^{2}+1954041 X Y+8703037 Y^{2}, \\
535437 X^{2}+408245 X Y+120801334 Y^{2}, \\
4413782 X^{2}+1926753 X Y+14855272 Y^{2} .
\end{array}
$$

It is not difficult to check that these forms are actually of order 5 and independent, e.g. by using the formulae for composition of quadratic forms as given by Shanks in [8].

Example 10 is, apart from example $\underline{7}$, the only field with prime discriminant that we encountered in our search; it occurred for $t=7 / 33$ (note that $7^{2}+7.33+33^{2}$ $=37^{2}$ ).

The fields 11 and 12 are listed since they are "irregular" for both 3 and 5: the 5 -rank of their class groups equals 3, while the 3-rank equals 2 . The fields 13 and 14 have class groups with unusual 5-primary parts; these groups are isomorphic to $C(5) \times C\left(5^{4}\right)$ and $C(5) \times C\left(5^{5}\right)$, respectively. We encountered these types of class groups only once. 
Finally two of the fields that Solderitsch found [10] are listed. These two fields have discriminants in the range of our computations; the absolute values of the discriminants of the other fields he found are much larger.

It is possible to do computations like these using other elliptic curves. However, if one uses elliptic curves that are defined over $\mathbf{Q}$, one cannot apply this method for $p \geqslant 11$, since rational $p$-torsion points on elliptic curves do not exist if $p \geqslant 11$. We did some computations for $p=7$, but did not succeed in finding new 7-rank $=3$ examples.

The computations of the class groups have been done using Shanks's algorithm as described in [8]. A feature of this algorithm is that it is theoretically possible that one does not compute the full class group, but that one only finds a subgroup of the class group; it is extremely unlikely that this occurred in our computations, but, strictly speaking, all the values of the 5-ranks we found are, in fact, lower bounds.

\begin{tabular}{|c|c|c|c|c|c|c|}
\hline$-\Delta(\mathrm{K})$ & & $t$ & $\mathrm{~h}$ & & & $L(1, x)$ \\
\hline 18397407 & 3.7 .876067 & $1 / 4$ & 2000 & $5 \times 5 \times 5$ & $2 \times 8$ & 1.465 \\
\hline 77778287 & 31.103 .24359 & $1 / 5$ & 6000 & $5 \times 5 \times 5$ & $2 \times 24$ & 2.137 \\
\hline 1156599359 & 47.67 .311 .1181 & $7 / 2$ & 34000 & $5 \times 5 \times 5$ & $2 \times 2 \times 68$ & 3.141 \\
\hline 2048074559 & 67.3323 .9199 & $2 / 7$ & 52500 & $5 \times 5 \times 25$ & $2 \times 42$ & 3.644 \\
\hline 7558314879 & 3.31 .883 .92041 & $7 / 4$ & 60000 & $5 \times 5 \times 25$ & $2 \times 4 \times 12$ & 2.168 \\
\hline 16704202367 & 47.109 .3260629 & $7 / 5$ & 125000 & $5 \times 5 \times 625$ & $2 \times 4$ & 3.038 \\
\hline 19283393759 & 7.19 .683 .21281 & $1 / 11$ & 173000 & $5 \times 5 \times 5$ & $2 \times 2 \times 346$ & 3.914 \\
\hline 39246913919 & 163.240778613 & $11 / 3$ & 219250 & $5 \times 5 \times 5$ & 1754 & 3.477 \\
\hline 69971761919 & 53.163 .8099521 & $3 / 11$ & 345000 & $5 \times 5 \times 25$ & $2 \times 276$ & 4.097 \\
\hline 116734226447 & 199.586604153 & $2 / 13$ & 305000 & $5 \times 5 \times 25$ & 488 & 2.804 \\
\hline 208703173647 & 3.67 .1038324247 & $5 / 11$ & 211000 & $5 \times 5 \times 5$ & $2 \times 844$ & 1.451 \\
\hline 222637549223 & prime & $7 / 33$ & 434625 & $5 \times 5 \times 5$ & 3477 & 2.894 \\
\hline 240820329839 & 223.2897 .372769 & $11 / 6$ & 579500 & $5 \times 5 \times 5$ & $2 \times 2318$ & 3.710 \\
\hline 315633202367 & 7.37 .1218661013 & $2 / 15$ & 579500 & $5 \times 5 \times 5$ & $2 \times 2318$ & 3.240 \\
\hline 338605831007 & 229.1478628083 & $5 / 12$ & 552500 & $5 \times 5 \times 25$ & 884 & 2.983 \\
\hline 407654485199 & 13.19 .199 .1021 .8123 & $11 / 7$ & 848000 & $5 \times 5 \times 5$ & $2 \times 2 \times 2 \times 848$ & 4.173 \\
\hline 440024496719 & 313.1405829063 & $16 / 3$ & 756000 & $5 \times 5 \times 5$ & 6048 & 3.580 \\
\hline 472440264519 & 3.199 .257 .311 .9901 & $11 / 26$ & 634000 & $5 \times 5 \times 5$ & $2 \times 2 \times 2 \times 634$ & 2.898 \\
\hline 477720858639 & 3.109 .863 .1692839 & $17 / 2$ & 700000 & $5 \times 5 \times 125$ & $2 \times 2 \times 56$ & 3.182 \\
\hline 488591920767 & 3.97 .269 .6241673 & $14 / 5$ & 357000 & $5 \times 5 \times 5$ & $2 \times 2 \times 714$ & 1.605 \\
\hline 526789501199 & 13.19 .2132751017 & $7 / 11$ & 991500 & $5 \times 5 \times 5$ & $2 \times 3966$ & 4.291 \\
\hline 719058505007 & 271.5527 .480071 & $10 / 9$ & 801000 & $5 \times 5 \times 5$ & $2 \times 3204$ & 2.968 \\
\hline 819641901567 & 3.53 .97 .401 .132529 & $5 / 14$ & 570000 & $5 \times 5 \times 25$ & $2 \times 2 \times 2 \times 114$ & 1.978 \\
\hline 825270838559 & 199.283 .1123 .13049 & $6 / 13$ & 1207000 & $5 \times 5 \times 5$ & $2 \times 2 \times 2414$ & 4.174 \\
\hline 1764613514207 & 379.3463 .1344491 & $15 / 7$ & 1004000 & $5 \times 5 \times 5$ & $2 \times 4016$ & 2.374 \\
\hline 2474580780719 & 463.1109 .4819357 & $1 / 21$ & 1884000 & $5 \times 5 \times 5$ & $2 \times 7536$ & 3.762 \\
\hline 3513582927119 & 487.7214749337 & $2 / 21$ & 2178000 & $5 \times 5 \times 5$ & $3 \times 5808$ & 3.650 \\
\hline 4025744542799 & 7.79 .401 .18154183 & $23 / 1$ & 1779000 & $5 \times 5 \times 5$ & $2 \times 2 \times 3558$ & 2.785 \\
\hline 6078086981679 & 3.181 .269 .41611637 & $19 / 7$ & 1751000 & $5 \times 5 \times 5$ & $2 \times 2 \times 3502$ & 2.231 \\
\hline 6822526267487 & 7.67 .14546964323 & $12 / 13$ & 2362500 & $5 \times 5 \times 125$ & $2 \times 378$ & 2.842 \\
\hline
\end{tabular}




\begin{tabular}{|c|c|c|c|c|c|c|}
\hline$-\Delta(K)$ & & t & $n$ & & & $L(1, x)$ \\
\hline 7111644846239 & 523.13597791293 & $17 / 9$ & 3797500 & $5 \times 5 \times 25$ & 6076 & 4.474 \\
\hline 7391579442047 & 499.1481 .2784453 & $7 / 18$ & 2030500 & $5 \times 5 \times 5$ & 16244 & 2.346 \\
\hline 8065721968127 & 19.31 .13693925243 & $20 / 7$ & 2720000 & $5 \times 5 \times 25$ & $2 \times 2176$ & 3.009 \\
\hline 9795957818927 & 7.73 .1873 .10235009 & $11 / 15$ & 2146000 & $5 \times 5 \times 5$ & $2 \times 2 \times 4292$ & 2.154 \\
\hline 10799568953999 & 7.79 .4093 .4771331 & $16 / 11$ & 4326000 & $5 \times 5 \times 5$ & $2 \times 2 \times 8652$ & 4.136 \\
\hline 13375918976399 & 7.79 .103 .3613 .64997 & $11 / 16$ & 3392000 & $5 \times 5 \times 5$ & $2 \times 2 \times 2 \times 3392$ & 2.914 \\
\hline 13598357713967 & 13.43 .24326221313 & $10 / 17$ & 3069000 & $5 \times 5 \times 5$ & $2 \times 12276$ & 2.615 \\
\hline 14077525107999 & 3.53 .199 .444914039 & $17 / 11$ & 3237000 & $5 \times 5 \times 5$ & $2 \times 2 \times 6474$ & 2.710 \\
\hline 15826902503327 & 661.7789 .3074063 & $20 / 9$ & 2877500 & $5 \times 5 \times 25$ & $2 \times 2302$ & 2.272 \\
\hline 16009647635519 & 419.787 .6917 .7019 & $27 / 2$ & 5085000 & $5 \times 5 \times 25$ & $2 \times 2 \times 2034$ & 3.993 \\
\hline 17124593400479 & 757.8363 .2704969 & $1 / 27$ & 4022000 & $5 \times 5 \times 5$ & $4 \times 8044$ & 3.053 \\
\hline 18178141409279 & 643.883 .32016791 & $18 / 11$ & 5274000 & $5 \times 5 \times 5$ & $2 \times 21096$ & 3.886 \\
\hline 18355577207519 & 613.29943845363 & $9 / 19$ & 6477750 & $5 \times 5 \times 5$ & 51822 & 4.750 \\
\hline 20434497658959 & 3.53 .211 .609094091 & $16 / 13$ & 3525000 & $5 \times 5 \times 125$ & $2 \times 2 \times 282$ & 2.450 \\
\hline 22226379018527 & 53.811 .14851 .34819 & $25 / 6$ & 3834000 & $5 \times 5 \times 5$ & $2 \times 2 \times 7668$ & 2.555 \\
\hline 22526019100319 & 7.109 .199 .419 .354073 & $3 / 26$ & 4190000 & $5 \times 5 \times 25$ & $2 \times 2 \times 2 \times 838$ & 2.773 \\
\hline 23031374411279 & 13.61 .46817 .620359 & $24 / 7$ & 6926000 & $5 \times 5 \times 5$ & $2 \times 2 \times 13852$ & 4.534 \\
\hline 23234046745007 & 7.103 .115663 .278609 & $5 / 24$ & 4260000 & $5 \times 5 \times 25$ & $2 \times 4 \times 852$ & 2.776 \\
\hline 23271228811967 & 691.33677610437 & $19 / 11$ & 2965250 & $5 \times 5 \times 5$ & 23722 & 1.931 \\
\hline 24008715204479 & 643.37338592853 & $11 / 18$ & 5808000 & $5 \times 5 \times 5$ & 46464 & 3.724 \\
\hline 24213534365039 & 53.103 .673 .6590677 & $8 / 21$ & 6842000 & $5 \times 5 \times 5$ & $2 \times 2 \times 13684$ & 4.368 \\
\hline 24307482796127 & 499.661 .73694993 & $9 / 20$ & 5068500 & $5 \times 5 \times 5$ & $2 \times 20274$ & 3.230 \\
\hline 29784718976207 & 13.67 .160009 .213713 & $1 / 29$ & 5702000 & $5 \times 5 \times 5$ & $2 \times 2 \times 11404$ & 3.282 \\
\hline 36573526186847 & 3.73 .163 .199 .499 .2381 & $28 / 5$ & 5264000 & $5 \times 5 \times 5$ & $2 \times 2 \times 2 \times 2 \times 2632$ & 2.735 \\
\hline 37262495315279 & 13.61 .46989275303 & $21 / 11$ & 7749000 & $5 \times 5 \times 5$ & $6 \times 10332$ & 3.988 \\
\hline 49985970332079 & 3.331 .50338338703 & $1 / 31$ & 6374000 & $5 \times 5 \times 5$ & $2 \times 25496$ & 2.832 \\
\hline 52508111150207 & 829.63339096683 & $20 / 13$ & 4877500 & $5 \times 5 \times 25$ & 7804 & 2.115 \\
\hline 54805390012079 & 7.151 .7517 .6897691 & $32 / 1$ & 7497000 & $5 \times 5 \times 5$ & $2 \times 2 \times 14994$ & 3.181 \\
\hline 55329101911439 & 103.823 .4723 .138197 & $19 / 14$ & 9997000 & $5 \times 5 \times 5$ & $2 \times 2 \times 19994$ & 4.222 \\
\hline 60410353317359 & 19.43 .53629 .1378763 & $17 / 16$ & 10724000 & $5 \times 5 \times 5$ & $2 \times 2 \times 21448$ & 4.335 \\
\hline 63123375138239 & 13.79 .103 .269 .2218351 & $2 / 31$ & 9944000 & $5 \times 5 \times 5$ & $2 \times 2 \times 2 \times 9944$ & 3.932 \\
\hline 69948783320639 & 1123.62287429493 & $33 / 1$ & 9438250 & $5 \times 5 \times 5$ & 75506 & 3.545 \\
\hline 76087582641167 & 19.61 .1699 .38639987 & $33 / 2$ & 8228000 & $5 \times 5 \times 5$ & $2 \times 4 \times 8228$ & 2.963 \\
\hline 76178156852447 & 13.73 .103 .9227 .84463 & $23 / 12$ & 8884000 & $5 \times 5 \times 5$ & $2 \times 2 \times 2 \times 8884$ & 3.198 \\
\hline 86754370349199 & 3.7 .43 .269 .357150557 & $11 / 23$ & 6098000 & $5 \times 5 \times 5$ & $2 \times 2 \times 2 \times 6098$ & 2.057 \\
\hline 93633351110319 & 3.379 .82351232287 & $29 / 8$ & 7569000 & $5 \times 5 \times 5$ & $2 \times 30276$ & 2.457 \\
\hline 102440524590047 & 7.157 .93212488253 & $27 / 10$ & 8060500 & $5 \times 5 \times 5$ & $2 \times 32242$ & 2.502 \\
\hline 109165179721247 & 13.53 .73 .883 .2457997 & $12 / 23$ & 8250000 & $5 \times 5 \times 625$ & $2 \times 2 \times 2 \times 66$ & 2.481 \\
\hline 133514240116127 & 13.103 .18329 .5440117 & $35 / 3$ & 11046000 & $5 \times 5 \times 5$ & $2 \times 2 \times 22092$ & 3.003 \\
\hline 143095169224847 & 7.53 .193 .1998452149 & $34 / 5$ & 7972000 & $5 \times 5 \times 5$ & $2 \times 4 \times 7972$ & 2.094 \\
\hline
\end{tabular}




\begin{tabular}{r|r|r|r|r|r|r}
$-\Delta(x)$ & & $t$ & $h$ & & & $L(1, x)$ \\
\hline 157897435920447 & 3.47 .433 .2586235499 & $2 / 35$ & 5090000 & $5 \times 5 \times 25$ & $2 \times 2 \times 2036$ & 1.273 \\
244367110736159 & 1483.164778901373 & $1 / 38$ & 19072750 & $5 \times 5 \times 5$ & 152582 & 3.833 \\
258559351511807 & 1171.1439 .153441403 & $14 / 25$ & 14785000 & $5 \times 5 \times 5 \times 5$ & $2 \times 11828$ & 2.889 \\
263424263462927 & 7.47 .223 .10993 .326617 & $39 / 1$ & 13962000 & $5 \times 5 \times 5$ & $2 \times 2 \times 2 \times 13962$ & 2.703 \\
317159735746287 & 3.7 .61 .199 .1244158873 & $11 / 29$ & 7008000 & $5 \times 5 \times 5$ & $2 \times 2 \times 2 \times 7008$ & 1.236
\end{tabular}

Two computer programs were used: one computes class groups of complex quadratic fields $K$ given their discriminants $\Delta(K)>-2.5_{10} 14$; the other is a double length version of this program [7]. All computations have been done on the CDC-computer system of SARA in Amsterdam.

Department of Mathematics

University of Leiden

Leiden. The Neitherlands

1. D. A. Buell, “Class groups of quadratic fields,” Math. Comp.. v. 30, 1976, pp. 610-623.

2. F. Diaz y Diaz, Sur le 3-Rang des Corps Quadratiques. Thèse de $3^{(0}$ cycle. Orsay, 1978.

3. F. Diaz y Diaz, D. Shanks \& H. C. Williams, "Quadratic fields with 3-rank equal to 4." Math. Comp., v. 33, 1979. pp. 836-840.

4. F. Diaz y Diaz, "Quelques discriminants irreguliers," Actas de las VII Jornadas Math.. HispanoLusas, Santander, Iunio 1979.

5. J. F. Mestre. Courbes Elliptiques et Groupes de Classes d'Ideaux de Certains Corps Quadratiques, Sém. de Théorie des Nombres, Bordeaux, 1979/1980. Exp. 15.

6. B. J. BIRCH \& W. KUYK (Editors), Modular Functions of One Variable IV. Lecture Notes in Math.. Vol. 476, Springer, Berlin, 1976.

7. R. J. Schoof, Two Algorithms for Determining Class Groups of Quadratic Fields, Report, Dept. of Math., University of Amsterdam. (In preparation.)

8. D. Shanks, Class Number, A Theory of Factorization and Genera, Proc. Sympos. Pure Math., Vol.20. Amer. Math. Soc., Providence, R.I., 1971, pp. 415-440.

9. D. Shanks \& P. Weinberger, “A quadratic field of prime discriminant requiring three generators for its class group and related theory," Acta Arith., v. 21, 1972, pp. 71-87.

10. J. J. SODERITSCH, "Quadratic fields with special class groups" (In preparation.) 